\title{
Intel Xeon Phi accelerated Weather Research and Forecasting (WRF) Goddard microphysics scheme
}

\author{
J. Mielikainen, B. Huang, and A. H.-L. Huang \\ Intel Parallel Computing Center, University of Wisconsin-Madison, Madison, Wisconsin, USA \\ Received: 23 October 2014 - Accepted: 17 November 2014 - Published: 12 December 2014 \\ Correspondence to: B. Huang (bormin@ssec.wisc.edu) \\ Published by Copernicus Publications on behalf of the European Geosciences Union.
}

\section{Abstract}

The Weather Research and Forecasting (WRF) model is a numerical weather prediction system designed to serve both atmospheric research and operational forecasting needs. The WRF development is a done in collaboration around the globe. Further5 more, the WRF is used by academic atmospheric scientists, weather forecasters at the operational centers and so on. The WRF contains several physics components. The most time consuming one is the microphysics. One microphysics scheme is the Goddard cloud microphysics scheme. It is a sophisticated cloud microphysics scheme in the Weather Research and Forecasting (WRF) model. The Goddard microphysics

10 scheme is very suitable for massively parallel computation as there are no interactions among horizontal grid points. Compared to the earlier microphysics schemes, the Goddard scheme incorporates a large number of improvements. Thus, we have optimized the Goddard scheme code. In this paper, we present our results of optimizing the Goddard microphysics scheme on Intel Many Integrated Core Architecture (MIC) hardware.

15 The Intel Xeon Phi coprocessor is the first product based on Intel MIC architecture, and it consists of up to 61 cores connected by a high performance on-die bidirectional interconnect. The Intel MIC is capable of executing a full operating system and entire programs rather than just kernels as the GPU does. The MIC coprocessor supports all important Intel development tools. Thus, the development environment is one familiar to a vast number of CPU developers. Although, getting a maximum performance out of MICs will require using some novel optimization techniques. Those optimization techniques are discussed in this paper. The results show that the optimizations improved performance of Goddard microphysics scheme on Xeon Phi 7120P by a factor of 4.7x. In addition, the optimizations reduced the Goddard microphysics scheme's share of 25 the total WRF processing time from 20.0 to $7.5 \%$. Furthermore, the same optimizations improved performance on Intel Xeon E5-2670 by a factor of $2.8 \times$ compared to the original code. 


\section{Introduction}

Scientific computing is moving towards the exascale era. In order to prepare legacy software to take full advantage of modern many-core supercomputing environments, the legacy code has to be modernized. This means taking a careful look at the code

5 modernization opportunities. Modern CPU cores contain several Single Instruction Parallel Data (SIMD) execution units for performing the same instruction on multiple data point simultaneously. Intel's Many Integrated Core Architecture (MIC) accelerator cards up the ante by having up to $61 \mathrm{CPU}$ cores with 16 SIMD execution units per core. Thus, both threading and vectorization techniques are explored in this paper. Furthermore,

10 code has to be optimized for frequent reuse of the cached data to increase computational intensity. This issue will also be explored in the paper. In addition, the Intel MIC coprocessor uses the same programming models and tools as processors. Thus, the optimization effort also benefits the code running on CPUs.

The Weather Research and Forecasting (WRF) is an open source Numerical

15 Weather Prediction (NWP) model. It is suitable for simulating weather phenomena ranging from meters to thousands of kilometers. WRF is the most widely used community weather forecast and research model in the world. Both operational forecasters and atmospheric researcher are using it in 153 countries. The WRF model contains two dynamics cores: the Non-hydrostatic Mesoscale Model core (Janjic, 2003) and the

20 Advanced Research WRF (Wang et al., 2008) core. A dynamic core contains a set of dynamic solvers that operates on a particular grid projection, grid staggering, and vertical coordinate system. The WRF model also contains several physics components, many of which can be used with both dynamic cores. The WRF has an extensible design. Therefore, it is possible to add physics, chemistry, hydrology models and other

25 features to it. In the real world scenarios WRF is initialized with boundary conditions and topography using observations. Thus, WRF is used in a variety of areas such as tropical storm prediction, simulation of wild fire, air-quality modeling, prediction of regional climate and storm-scale research.

8943

WRF software architecture takes advantage of the distributed and shared memory systems. However, vector capabilities of Xeon Phi coprocessors and the latest CPUs are only used by the WSM5 microphysics scheme. The other modules have not been vectorized at all. In this paper, we present our results of vectorizing and further op5 timizing another microphysics scheme, called Goddard scheme, on the current Xeon Phi. Furthermore, with the advent of unified AVX-512 vector extension instructions on Intel's future Knights Landing MIC and Skylake CPU, utilization of vector processing is becoming essential for a peak performance on data parallel programs such as WRF. Meanwhile, OpenMP 4.0 offers important solutions for vectorization is a standard way. 10 These will make our optimization work imperative for optimal performance of the WRF on any future Intel hardware.

Although, Intel Xeon Phi is a new product there are already exists of a lot of publications characterizing its performance. A case study of the WRF on Xeon Phi analyzed the scalability trade-offs running one or more ranks of an MPI program on an Intel

15 MIC chip (Meadows, 2012). Currently, the latest community release (WRFV3.6) runs natively on Xeon Phi. However, only WSM5 microphysics scheme has been fully optimized to take full advantage of the Intel MIC (Michalakes, 2013). WSM5 contains 1800 lines of code out of an approximately 700000 in WRF. WSM5 is just one of over ten microphysics schemes in WRF. There are also six other physics components in WRF

20 for a total of seven physics components. In addition, there is also a dynamics core in WRF that has not been optimized for Xeon Phi yet. This paper describes our efforts to optimize Goddard microphysics scheme to take advantage of the Intel's MIC. This represents our first step towards fully acceleration WRF on Intel MIC.

Other examples of Intel MIC acceleration are leukocyte tracking using medical

25 imaging techniques (Fang et al., 2014), real-world geophysical application (Weinberg et al., 2014) and an astrophysics package (GADGET), which is used for cosmological $\mathrm{N}$-body/SPH simulations to solve a wide range of astrophysical tasks (Borovska and Ivanova, 2014). Other successful examples of using Intel MIC for accelerated computing are multiple sequence alignment software (Borovska et al., 2014), atomistic simula- 
tion package (Reida and Bethunea, 2014) and general purpose subroutine system for the numerical solution of the incompressible Navier-Stokes equations (Venetis et al., 2014). In addition, the use of Intel MIC for the computation of the partial correlation coefficient with information theory (PCIT) method for detecting interactions between 5 networks has also been explored (Stanzione, 2014). Furthermore, the UCD-SPH utilizing the Smoothed Particle Hydrodynamics (SPH) method for modelling wave interaction with an Oscillating Wave Surge Converter (OWSC) device has been optimized for Intel MIC (Lalanne, 2014). Moreover, the performance of Intel MIC vs. CPU has been studied using a parallel simulation of high-dimensional American option pricing

10 (Hu et al., 2014). A fast short-read aligner has also been implemented on Intel MIC (Chan et al., 2014).

WRF modules have a relatively low arithmetic intensity and limited operand reuse. Furthermore, large working set size of WRF modules overflows cache memories of the current coprocessors. Therefore, WRF optimization process will start by modifying the

15 code to expose vectorization and reducing the memory footprint size for better cache utilization. Techniques such as less-than-full-vector loop vectorization have to be used for a peak performance (Tian et al., 2013).

A nice advantage of using Xeon Phi for WRF acceleration is the fact that an improvement in the whole WRF can be observed after each optimization step. This is due to WRF running on a Xeon Phi's native mode. This is one huge advantage of Xeon Phi compared to the GPUs, which we have been using for WRF optimization earlier. Just like Xeon Phi's offload mode, an individual GPU kernel needs to transfer data between CPU and GPU. The data transfer overhead for a WRF module on a GPU is usually enough to kill any potential speedup for the whole WRF. Only when the whole codebase has been translated to run on GPU can WRF get any speedup on GPUs. For Xeon Phis, a speedup can be observed from day one.

In Sect. 2, we review the main characteristics of the Goddard microphysics scheme. Section 3 introduces MIC hardware characteristics. Results of the code optimization of

the Goddard scheme are given in Sect. 4. Conclusion and plans for future work are described in Sect. 5.

\section{Goddard microphysics scheme}

The WRF physics components are microphysics, cumulus parameterization, planetary 5 boundary layer (PBL), land-surface/surface-layer model and short-/longwave radiation. WRF physics components and their interactions are shown in Fig. 1. Dynamics core and its model integration procedure are shown in Fig. 2.

The Goddard microphysics scheme was introduced in (Tao and Simpson, 1993). The microphysical scheme has been modified to reduce the over-estimated and unrealistic 10 amount of graupel in the strati form region (Tao et al., 2003 and Lang et al., 2007). In addition, saturation issues have been addressed better (Tao et al., 2003) and more realistic ice water contents for longer-term simulations have been obtained (Zeng, 2008, 2009). The microphysical processes simulated in the model are demonstrated in Fig. 3 and explained in Table 1. The process is a single moment bulk cloud microphysics 15 scheme, which update 7 variables, (deviation of potential temperature, water vapor and mixing ratio of 5 hydrometeors (cloud water, cloud ice, rain water, snow, graupel/hail) in every time step using prognostic equation saturation process and microphysical interaction process.

\section{Intel MIC}

Intel MIC can be programmed using either offload or native programming model. Compiler directives can be added to the code to mark regions of the region of the code that should be offloaded to the Intel Xeon Phi coprocessor. This approach is familiar to the users of Graphics Processing Units (GPU)s as computational accelerators. Instead of using this cumbersome and error-prone approach we used the native pro25 gramming model of Intel MIC for our work. In the native mode, the code is compiled on 
the host using Intel's compiler with the compiler switch -mmic to generate code for the $\mathrm{MIC}$ architecture. After that, the resulting binary is copied to the MIC coprocessor and the binary is executed there. This makes code porting process straightforward, but the code optimization still takes skill and effort. Furthermore, the latest community release

5 of WRF (WRF v3.6) already runs natively on Xeon Phi. So, we are taking the current Intel MIC WRF code as a baseline and performing optimizations to individual modules in the WRF code. In this paper code optimization results for Goddard microphysics scheme will be presented.

Intel Xeon Phi coprocessor 7120P, which we used for benchmarks in the paper, 10 contains 61 independent cores running at a low frequency of $\sim 1.2 \mathrm{GHz}$. Each core supports four-way hyperthreading for 244 concurrent threads running of the Intel Xeon Phi. Inside each core there is a dedicated vector unit using 512-bit vector registers for Single Instruction Multiple Data (SIMD) operations. The SIMD instruction set supports data parallel execution of floating point multiplication, addition, fused multiply-add 15 and division, exponential functions, logarithms, trigonometric functions, bitwise logical functions, gather/scatter memory access operations. Bit-masked operations for most operations are also provided (Rahman, 2013).

Furthermore, Intel Xeon Phi has 16 GB of high bandwidth Graphics Double Data Rate (GDDR5) memory. Unified L2 caches in each core are used to cache the access 20 to GDDR5 memory. In addition, each core has L1 cache both for instruction and data, with a typical access time of 1 cycle. The caches are fully coherent and implement x86 memory order model. The memory bandwidth provided by L1 and L2 caches is 15 and 7 times faster than GDDR5 bandwidth. Therefore, effective use of caches is the optimization feature for achieving the peak performance of Intel Xeon Phi (Chrysos, 25 2014).

Summary of the specifications of the Intel Xeon Coprocessor 7120P is shown in Table 2. More details of Intel MIC programming can be found in (Jeffers and Reinders, 2013). A dual socket octa-core Intel Xeon E5-2670 CPU was also used for bench-

marking the performed Goddard code optimizations. The specifications of the CPU are shown in Table 3.

\section{Code optimization}

To test Goddard scheme on MIC we used a CONtinental United States (CONUS) 5 benchmark data set for $12 \mathrm{~km}$ resolution domain for 24 October 2001. A WRF domain is a geographic region of interest discretized into a 2-dimensional grid parallel to the ground. Each grid point has multiple levels, which correspond to various vertical heights in the atmosphere. The size of the CONUS $12 \mathrm{~km}$ domain is $433 \times 308$ horizontal grid points with 35 vertical levels. As shown in Fig. 4, the test problem is a $12 \mathrm{~km}$ resolution

$1048 \mathrm{~h}$ forecast over the Continental US capturing the development of a strong baroclinic cyclone and a frontal boundary that extends from north to south across the entire US Goddard microphysics computation is organized into a 3-dimensional grid where the microphysical process computations in each column ( $k$-dimension) are processed independently. Thus, there are two dimensions of parallelism ( $i$ - and $j$-dimensions)

15 to work with. We used Intel's Fortran compiler, ifort, version 15.0.0 for compiling the Goddard source code for both Intel Xeon E5-2670 CPU and Intel Xeon Phi 7120P coprocessor. For CPU compilation the following default WRF compiler options for ifort were used: -O3 -openmp -w -ftz -fno-alias -auto -fp-model fast = 1 -no-prec-div -noprec-sqrt -auto -align array64byte. For Intel MIC the following compiler options from the WRF v3.6 distribution were used: -mmic -auto -ftz -fno-alias -fimf-precision = low -fimf-domain-exclusion = 15 -opt-assume-safe-padding -opt-streaming-stores always -opt-streaming-cache-evict $=0$-mP2OPT_hlo_pref_use_outer_strategy = F -fp-model fast = 1 -no-prec-div -no-prec-sqrt -auto -align array64byte. Dynamic scheduling was used for OpenMP work sharing construct in a do-loop.

As can be seen from Fig. 5, the microphysics is the most time consuming physics component in the WRF. Goddard microphysics scheme has 2355 lines of code compared to over 60000 lines of code in the ARW dynamics code. In total, the WRF con- 
sists of over 500000 lines of code. Therefore, it makes sense to optimize Goddard microphysics scheme first as this relatively small module can still give an overall speedup to the whole WRF. A summary of the various optimization steps is shown in Fig. 6. The processing times are an average of 10 execution runs. These processing times 5 are measured running a stand-alone driver for the Goddard scheme. A default domain decomposition of 4 horizontal and 61 vertical tiles were used by the first version of Goddard code (v1).

\subsection{Removal of the vertical dimension from temporary vectors for reduced memory footprint}

10 The first optimization step (v2) was modifying the dimensions of some intermediate variables so that the $k$-dimension of that variable is removed. This process can be performed for variables that only require the use on one $k$-dimension value at a time. This optimization had the effect of reusing the cached data to increase computational intensity. An example of this process is shown in Fig. 7. The processing time was 15 reduced from 223.0 to $206.4 \mathrm{~ms}$.

\subsection{Loop transformations to enable vectorization of affected loops}

Vectorizing the main subroutine, saticel_s, was done in preparation of the next major optimization step. Vectorization refers to the process where a scalar implementation, which does an operation one pair of operands at a time, is converted to a vector pro20 cess, where a single instruction can refer to a vector. This adds a form of parallelism to software in which one instruction or operation is applied to multiple pieces of data. The benefit is more efficient data processing and improved code performance.

In this optimization step, an $i$-loop nested inside $j$ - and /-loops were split into nine separate $i$-loops. This is illustrated in Fig. 8 . This allows the compiler to vectorize those

25 innermost loops in $i$-dimension.

8949

\subsection{Collapse $i$ - and $j$-loops into smaller cells for smaller footprint per thread}

In the next optimization step (v3) input data is copied to vector sized arrays of 16 elements. Each thread will process only 16 data elements. Thus, better data locality is achieved as more of the data can be accessed from the cache memories. In Fig. 9,

5 Goddard multithreaded code for this technique is shown. The drawback of this method is the overhead of copying input data to temporary arrays. Similarly, output data have to be copied from vector size arrays into to the original big arrays. Dynamic scheduling was used for OpenMP work sharing construct in the do-loop. The actual computation is performed in the main Goddard subroutine, saticel_s, 16 grid points at a time.

\section{$10 \quad 4.4$ Addition of vector alignment directives}

In the code version (v4) vector alignment directives were added before each $i$-loop. So, that the compiler is able to make optimizations for the data access. Data alignment is a method to force the compiler to create data objects in memory on specific byte boundaries. This is done to increase efficiency of data loads and stores to and from the 15 processor. This had a bigger effect of the code running on CPU than on MIC.

\subsection{Additional optimization of temporary variables for reduced memory footprint}

In v6, we modified the dimensions of some intermediate variables so that the $k$ dimension of that variable is removed. This process can be performed for variables 20 that only require the use on one $k$-dimension value at a time. For the CONUS $12 \mathrm{~km}$ data the vertical dimension is 35 . So, the memory saving can be significant. This facilitates better use of cache memory for faster data access. In total, 9 temporary variables had their dimension reduced from two to one. This reduced the processing time is from 107.3 to $101.3 \mathrm{~ms}$. 


\subsection{Code validation}

Code validation was performed by checking that all code versions produced the same output values on CPU. During code validation precise math compiler options were used. This tells the compiler to strictly adhere to value-safe optimizations when im-

5 plementing floating-point calculations. It disables optimizations that can change the result of floating-point calculations, which is required for strict ANSI conformance. These semantics ensure the reproducibility of floating-point computations, including code vectorized or auto-parallelized by the compiler. However, this compiler options slows down the performance. Therefore, the WRF does not use this compiler option as a default 10 option.

This is in stark contrast to a situation when the default WRF compiler options are used, which tells the compiler to use more aggressive optimizations when implementing floating-point calculations. These optimizations increase speed, but may affect the accuracy or reproducibility of floating-point computations.

\subsection{Effects of the code optimization on CPU performance}

Figure 10 shows the effect of the compiler optimizations on CPU performance. The overall speedup from the code optimization on an Intel Xeon E5-2670 CPU was 2.8x compared to $4.7 \times$ on the Intel Xeon Phi 7120P. The fact that the same code optimizations also improved CPU performance indicates that the single source code for both

20 CPU and MIC is a reality. This reduces development costs as any programming work done to modernize WRF for MICs will also improve its performance on legacy CPUs.

\subsection{Analysis using instruction mix report from Intel Software Development Emulator}

We used Intel Software Development Emulator to get a count of the number of exe-

cuted. Figure 11 shows the total number of instructions executed for both the original

8951

and optimized Goddard source code. Instruction counts are shown for AVX, AVX2 and AVX512 instructions sets Instruction count reduction for AVX512 instruction set is quite small for the original code due to limited vectorization of the code. However, the optimized code has a lower instruction count because of the use of vector instructions 5 instead of scalar instructions. Furthermore, AVX512 offers significantly improved code vectorization capabilities as is evident from its low instruction count compared to AVX and AVX2 instruction counts on the optimized code.

\subsection{The best processing times both on one and two socket CPU configurations and Intel MIC}

10 In Fig. 12, a summary of total elapsed times for execution of Gorrard calculation on Xeon CPU with 1 socket and 2 socket configurations and on Intel MIC. The results show that the scaling from a single CPU socket to a dual socket configuration is less than optimal due to memory bound nature of the code. Before the code optimization was performed, a dual socket CPU was faster than MIC. However, after the optimiza-

15 tion process the performance of MIC is even higher than a dual socket CPU configuration. Furthermore, the relative performance increase from the code optimizations was higher for the Intel MIC than CPUs. Thus, the performed optimizations are even more important on MIC due to its larger speedup benefit from those optimizations.

\subsection{Performance effects of multi-threading and vectorization}

20 In Fig. 13, effects of orthogonal optimization techniques of multi-threading and vectorization on 1 socket Xeon CPU configurations and on Intel MIC are shown. Unoptimized original code was used for scalar performance on the figure. That required adding -no-vec compiler option for Intel's Fortran compiler to disable code vectorization. Utilizing both multi-threading and vectorization was a key to a good performance both on CPU and MIC platforms. In the end, Intel Xeon Phi coprocessor were able to reach 
heights in performance beyond that of an Intel Xeon processor, but it required both multi-threading and vectorization to achieve that.

\subsection{Performance effects of the optimization of Goddard microphysics scheme on the WRF}

5 The optimized Goddard microphysics code was incorporated back into the WRF. Intel's VTune profiles measurements in Fig. 14, show that after code optimizations Goddard scheme takes only $7.6 \%$ of the total processing time and dynamics is even more dominant at $71.5 \%$ share of the total processing time. Thus, it is a natural candidate for further optimization of the WRF. In addition, both radiative transfer and planetary

10 boundary layer are also two physics categories that warrant further optimization efforts as they both take over $7 \%$ of the total processing time.

\section{Conclusions}

We have shown in this paper that Intel MIC can be faster than a dual socket CPU system. However, to achieve that, both multi-threading and vectorization have to uti-

15 lized. Furthermore, code has to be optimized for frequent reuse of the cached data to increase computational intensity. Following there guidelines, the optimization of the WRF Goddard microphysics scheme was described in this paper.

The results show that the optimizations improved performance of Xeon Phi 7120P by $4.7 \times$. This translates into $1.15 \times$ increase in total WRF processing time. Furthermore, 20 the optimizations improved performance on Intel Xeon E5-2670 by a factor of $2.8 \times$. The optimizations that were performed were quite generic in nature. Those optimizations included vectorization of the code to utilize vector units inside each CPU. Furthermore, memory access was improved by optimizing the access to some of intermediate data arrays.

8953

The generic nature of the performed optimizations means that the optimized code will run efficiently on future Intel MICs and CPU. The next generation Intel MIC, Intel Knights Landing (KNL), is not fully compatible with the previous generation, since AVX-512 SIMD instructions on KNL are encoded differently than the Larrabee New 5 Instructions (LRBni). However, it is expected that the optimization that we have performed so far on the code will also run well on KNL. The future Intel CPUs, such as Skylake-EX, will also use the AVX-512 instruction encoding for vector instructions.

The Goddard microphysics scheme optimization work represents an initial step towards fully vectorizing the WRF. As could be seen from the speedup figures, optimiza10 tion of the code is essential for a good performance on the MIC and modern CPUs. Thus, the work will continue in the optimization of the other WRF modules.

Acknowledgements. This work is supported by Intel Corporation under Grant No. MSN171479 to establish the Intel Parallel Computing Center at the University of Wisconsin-Madison. The authors would like to thank Bob Burroughs and Michael Greenfield for generous support.

\section{References}

Borovska, P. and Ivanova, D.: Code Optimization and Scaling of the Astrophysics Software Gadget on Intel Xeon Phi, Partnership for Advanced Computing in Europe (PRACE), 136 pp., 2014.

Borovska, P., Gancheva, V., and Tsvetanov, S.: Optimization and Scaling of Multiple Sequence 20 Alignment Software ClustalW on Intel Xeon Phi, Partnership for Advanced Computing in Europe (PRACE), 138 pp., 2014.

Chan, S. H., Cheung, J., Wu, E., Wang, H., Liu, C., M. Zhu, X., and Lam, T. W.: MICA: a fast short-read aligner that takes full advantage of Intel Many Integrated Core Architecture (MIC), arXiv preprint, 1402, 2014.

25 Chrysos, G.: Intel ${ }^{\circledR}$ Xeon Phi ${ }^{\mathrm{TM}}$ Coprocessor - the Architecture, Intel Whitepaper, 2014.

Fang, J., Sips, H., Zhang, L., Xu, C., Che, Y., and Varbanescu, A. L.: Test-driving Intel Xeon Phi, in: Proceedings of the 5th ACM/SPEC International Conference on Performance Engineering, ACM, 137-148, 2014. 
Hu, Y., Li, Q., Cao, Z., and Wang, J.: Parallel simulation of high-dimensional American option pricing based on CPU versus MIC, in: Concurrency and Computation: Practice and Experience, 2014.

Janjic, Z. I.: A nonhydrostatic model based on a new approach, Meteorol. Atmos. Phys., 82, 271-285, 2003.

Jeffers, J. and Reinders, J.: Intel Xeon Phi Coprocessor High Performance Programming, Morgan-Kauffman, Newnes, 2013.

Koesterke, L., Koltes, J. E., Weeks, N. T., Milfeld, K., Vaughn, M. W., Reecy, J. M., and Stanzione, D.: Discovery of biological networks using an optimized partial correlation coefficient with information theory algorithm on Stampede's Xeon and Xeon Phi processors, in: Concurrency and Computation: Practice and Experience, 2014.

Lalanne, C., Rafiee, A., Dutykh, D., Lysaght, M., and Dias, F. L.: Enabling the UCD-SPH code on the Xeon Phi, Partnership for Advanced Computing in Europe (PRACE), 131 pp., 2014.

Lang, S., Tao, W.-K., Cifelli, R., Olson, W., Halverson, J., Rutledge, S., and Simpson, J.: Improv-

15 ing simulations of convective system from TRMM LBA: easterly and Westerly regimes, J. Atmos. Sci., 64, 1141-1164, 2007.

Meadows, L.: Experiments with WRF on intel ${ }^{\circledR}$ Many Integrated Core (Intel MIC) Architecture, OpenMP in a Heterogeneous World, 130-139, 2012.

Michalakes, J.: Optimizing Weather Models for Intel Xeon Phi, Intel Theater Presentation SC '13, 20 November 2013.

Rahman, R.: Intel ${ }^{\circledR}$ Xeon Phi ${ }^{\mathrm{TM}}$ Coprocessor Architecture and Tools: the Guide for Application Developesr, Apress, 2013.

Reida, F. and Bethunea, I.: Optimising CP2K for the Intel Xeon Phi, Partnership for Advanced Computing in Europe (PRACE), 140 pp., 2014.

25 Skamarock, W. C., Klemp, J. B., Dudhia, J., Gill, D. O., Barker, D. M., Duda, M. G., Huang, X., Wang, W., and Powers, J. G.: A description of the Advanced Research WRF Version3, NCAR Technical Note, TN-4475+STR, Boulder, Colorado, USA, 2008.

Tao, W. K. and Simpson, J.: The Goddard cumulus ensemble model, Part I: Model description, Terr. Atmos. Ocean. Sci., 4, 35-72, 1993.

so Tao, W.-K., Simpson, J., Baker, D., Braun, S., Chou, M. D., Ferrier, B., and Wetzel, P.: Microphysics, radiation and surface processes in the Goddard Cumulus Ensemble (GCE) model, Meteorol. Atmos. Phys., 82, 97-137, 2003.

8955

Tian, X., Saito, H., Preis, S. V., Garcia, E. N., Kozhukhov, S. S., Masten, M., Cherkasov, A. G., and Panchenko, N.: Practical SIMD vectorization techniques for Intel ${ }^{\circledR}$ Xeon Phi coprocessors, in: IEEE 27th International Parallel and Distributed Processing Symposium Workshops \& PhD Forum (IPDPSW), 1149-1158, 2013.

5 Venetis, I. E., Goumas, G., Geveler, M., and Ribbrock, D.: Porting FEASTFLOW to the Intel Xeon Phi: Lessons Learned, Partnership for Advanced Computing in Europe (PRACE), 139 pp., 2014.

Weinberg, V., Allalen, M., and Brietzke, G.: Towards Porting a Real-World Seismological Application to the Intel MIC Architecture, Partnership for Advanced Computing in Europe (PRACE), 141 pp., 2014.

Zeng, X., Tao, W.-K., Lang, S., Hou, A., Zhang, M., and Simpson, J.: On the sensitivity of Atmospheric ensemble to cloud microphysics in long-term cloud-resolving model simulations, J. Meteor. Soc. Jpn. A, 86, 45-65, 2008.

Zeng, X., Tao, W.-K., Zhang, M., Hou, A. Y., Xie, S., Lang, S., Li, X., Starr, D., Li, X., and

15 Simpson, J.: The indirect effect of ice nuclei on atmospheric radiation, J. Atmos. Sci., 66, 41-61, 2009. 
Table 1. Key to Fig. 3.

\begin{tabular}{ll}
\hline Symbol & Meaning \\
\hline$P_{\mathrm{IMLT}}$ & Melting of cloud ice to form cloud water. \\
$P_{\mathrm{IDW}}$ & Depositional growth of cloud ice at expense of cloud water. \\
$P_{\mathrm{IHOM}}$ & Homogeneous freezing of cloud water to form cloud ice. \\
$P_{\mathrm{IACR}}$ & Accretion of rain by cloud ice; produces snow or graupel depending on the amount \\
& of rain. \\
$P_{\mathrm{RACl}}$ & Accretion of cloud ice by rain; produces snow or graupel depending on the amount \\
& of rain. \\
$P_{\mathrm{RAUT}}$ & Auto-conversion of cloud water to form rain. \\
$P_{\mathrm{RACW}}$ & Accretion of cloud water by rain. \\
$P_{\mathrm{REVP}}$ & Evaporation of rain. \\
$P_{\mathrm{RACS}}$ & Accretion of snow by rain; produces graupel if rain or snow exceeds threshold. \\
$P_{\mathrm{SACW}}$ & Accretion of cloud water by snow. Also enhances snow melting. \\
$P_{\mathrm{SACR}}$ & Accretion of rain by snow. Produces graupel if rain or snow exceeds threshold; if \\
& not, produces snow. Also, the accreted water enhances snow melting. \\
$P_{\mathrm{SACl}}$ & Accretion of cloud ice by snow. \\
$P_{\mathrm{SAUT}}$ & Auto-conversion (aggregation) of cloud ice to form snow. \\
$P_{\mathrm{SFW}}$ & Bergeron process (deposition and riming)-transfer of cloud water to form snow. \\
$P_{\mathrm{SFI}}$ & Transfer rate of cloud ice to snow through growth of Bergeron process embryos. \\
$P_{\mathrm{SDEP}}$ & Depositional growth of snow. \\
$P_{\mathrm{SSUB}}$ & Sublimation of snow. \\
$P_{\mathrm{SMLT}}$ & Melting of snow to form rain. \\
$P_{\mathrm{GAUT}}$ & Auto-conversion (aggregation) of snow to form graupel. \\
$P_{\mathrm{GFR}}$ & Probabilistic freezing of rain to form graupel. \\
$P_{\mathrm{GACW}}$ & Accretion of cloud water by graupel. \\
$P_{\mathrm{GACl}}$ & Accretion of cloud ice by graupel. \\
$P_{\mathrm{GACR}}$ & Accretion of rain by graupel. \\
$P_{\mathrm{GACS}}$ & Accretion of snow by graupel. \\
$P_{\mathrm{GSUB}}$ & Sublimation of graupel. \\
$P_{\mathrm{GMLT}}$ & Melting of graupel to form rain. \\
$P_{\mathrm{GWET}}$ & Wet growth of graupel. \\
&
\end{tabular}

8957

Table 2. Specifications of the Intel Xeon Coprocessor 7120P.

\begin{tabular}{|c|c|}
\hline Hardware attribute & Value \\
\hline Number of cores & 61 \\
\hline Frequency of cores & $1.238 \mathrm{GHz}$ \\
\hline GDDR5 memory size & $16 \mathrm{~GB}$ \\
\hline Number of hardware threads per core & 4 \\
\hline SIMD vector registers & 32 (512-bit wide) per thread context \\
\hline Flops/cycle & 16 (double precision), 32 (single precision) \\
\hline $\begin{array}{l}\text { Theoretical peak performance } \\
\text { L2 cache per core }\end{array}$ & $\begin{array}{l}1 \text { TFlops s}^{-1} \text { (double precision), } 2 \text { TFlops s }^{-1} \text { (single precision) } \\
512 \mathrm{~KB}^{2}\end{array}$ \\
\hline L1 cache per core & $32 \mathrm{~KB}$ instruction cache $/ 32 \mathrm{~KB}$ data cache \\
\hline
\end{tabular}


Table 3. Specifications of the Intel Xeon E5-2670.

\begin{tabular}{ll}
\hline Hardware attribute & Value \\
\hline Number of cores & 8 \\
Number of threads & 16 \\
Clock speed & $2.6 \mathrm{GHz}$ \\
SIMD vector registers & $18(256-$ bit wide) per thread context \\
Flops/cycle & 4 (double precision), 8 (single precision) \\
Theoretical peak performance & $167 \mathrm{GFlops}^{-1}$ (double precision), 333 GFlops ${ }^{-1}$ (single precision) \\
L3 cache & $20 \mathrm{MB}$ \\
L2 cache per core & $256 \mathrm{~KB}$ \\
L1 cache per core & $32 \mathrm{~KB}$ \\
\hline
\end{tabular}

8959

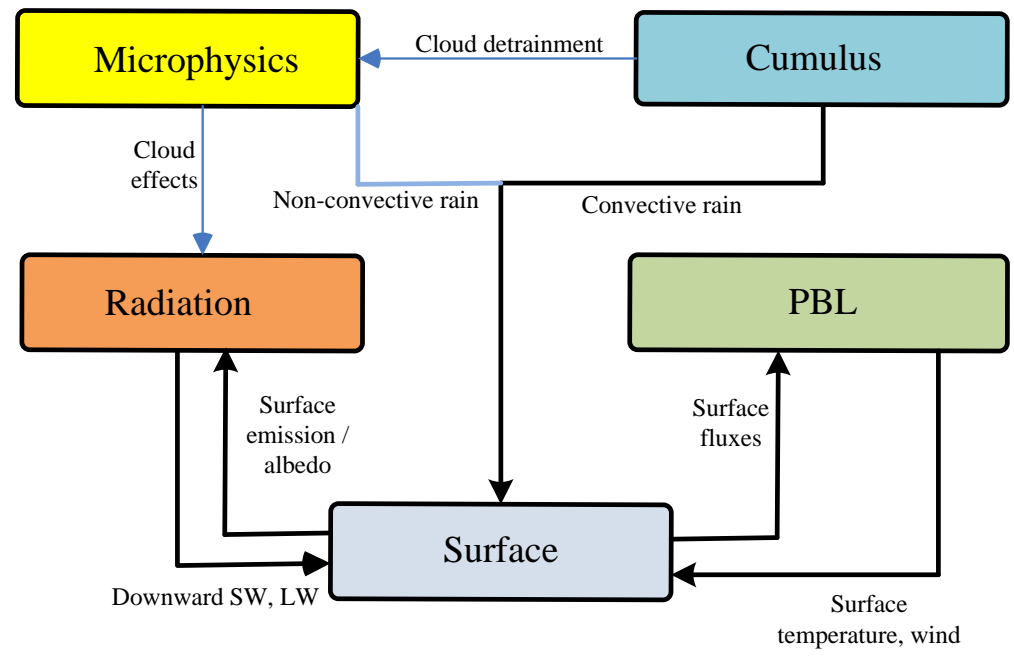

Figure 1. WRF physics components are microphysics, cumulus parametrization, planetary boundary layer (PBL), land-surface/surface-layer model, shortwave (SW) and longwave (LW) radiation. 
Begin time step

Runge-Kutta (RK) Loop (steps 1, 2 and 3)

(i) Advection, p-grad, buoyancy

(ii) Physics if step 1

(iii) Mixing, other non-RK dynamics

(iv) Assemble dynamics tendencies

Acoustic step loop

(i) Advance $\mathrm{U}, \mathrm{V}$ then $\mathrm{m}, \mathrm{Q}$ then $\mathrm{W}, \mathrm{f}$

(ii) Time-average $\mathrm{U}, \mathrm{V}$ and $\mathrm{W}$

End acoustic loop

Advance scalars using time-averaged $\mathrm{U}, \mathrm{V}$ and $\mathrm{W}$

End Runge-Kutta loop

Adjustement physics (microphysics)

End time step

Figure 2. WRF model integration procedure.

8961

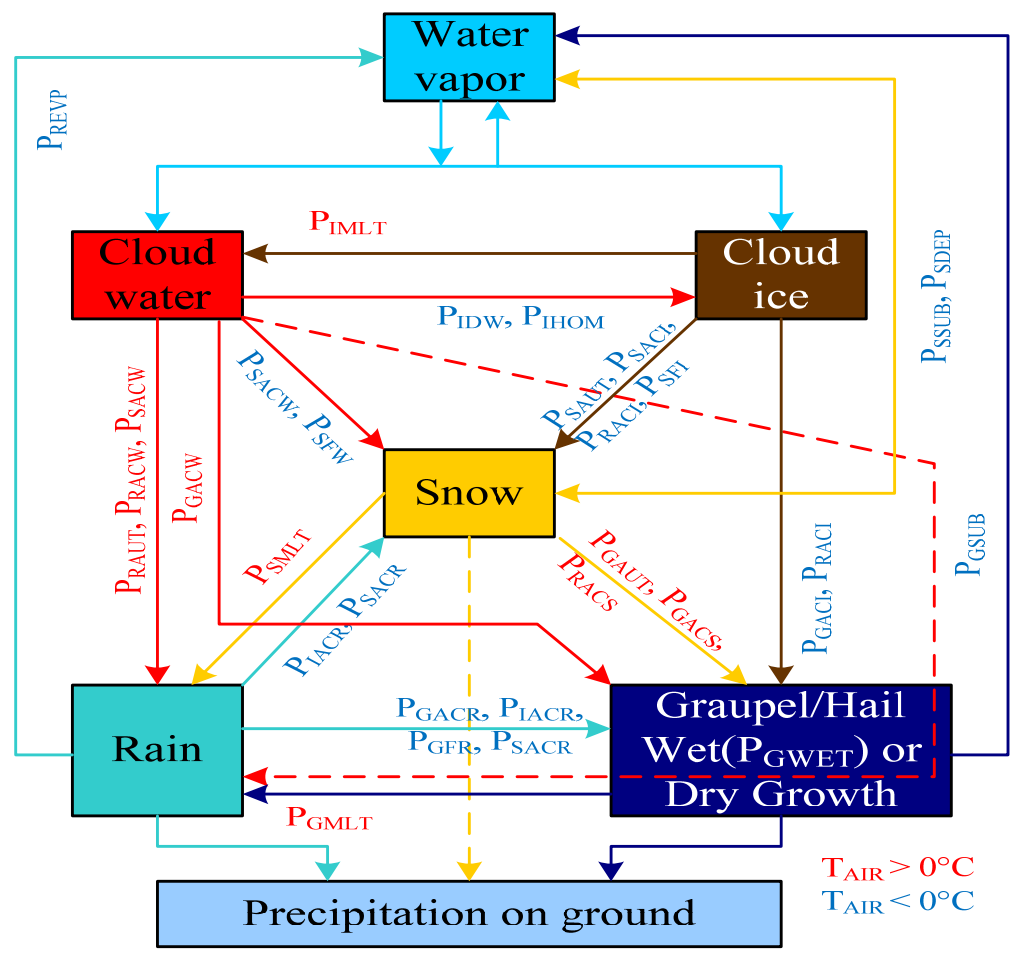

Figure 3. Cloud physics process simulated in the model with the snow field included. See Table 1 for an explanation of the symbols. 


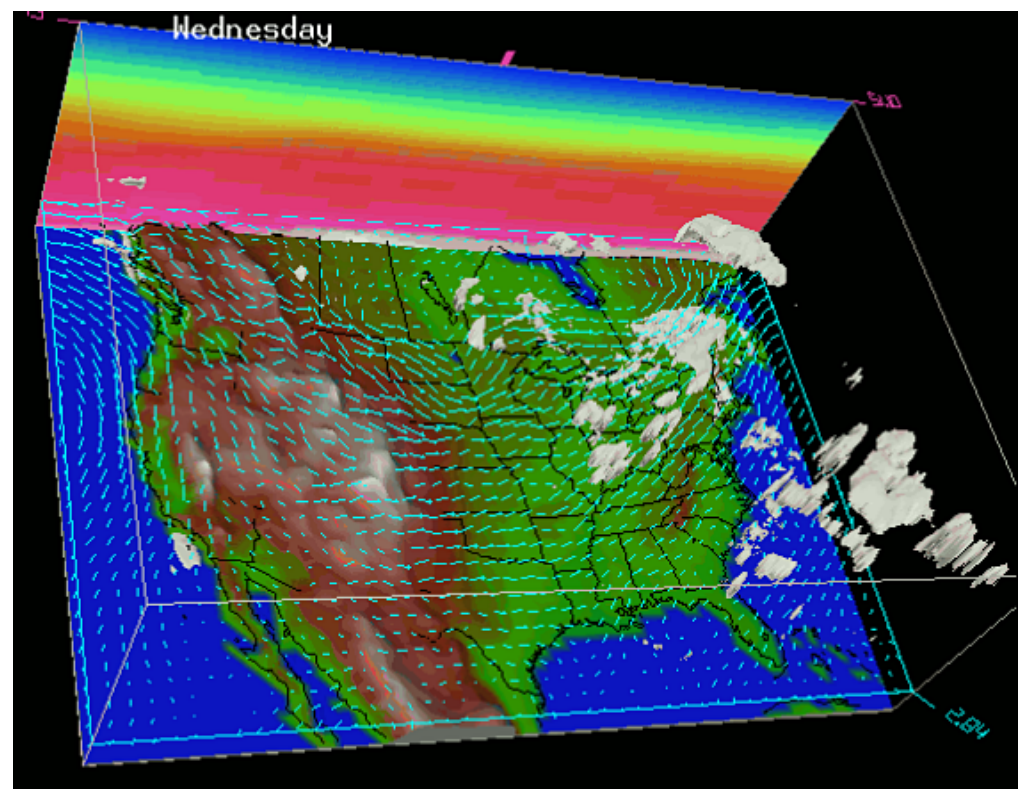

Figure 4. CONUS $12 \mathrm{~km}$ domain.

8963

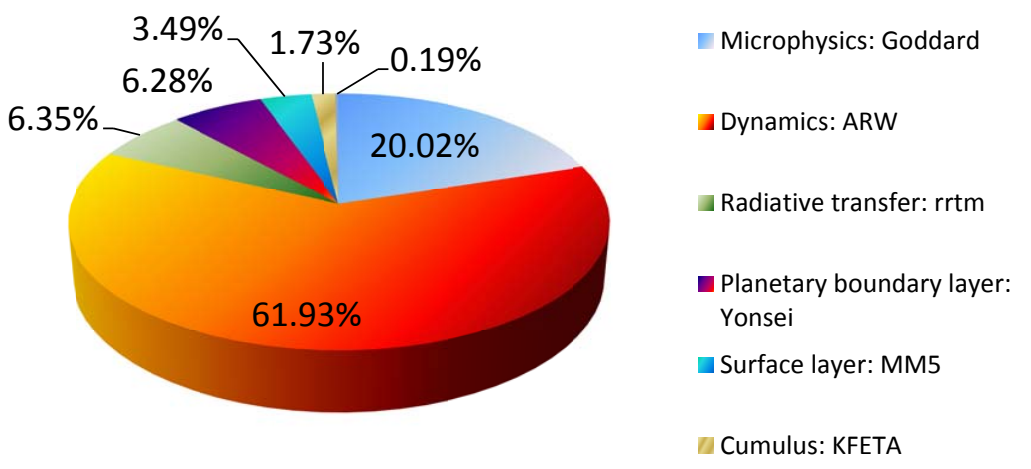

Figure 5. MIC processing times for the original WRF code as measured by Intel's profiling tool VTune. 


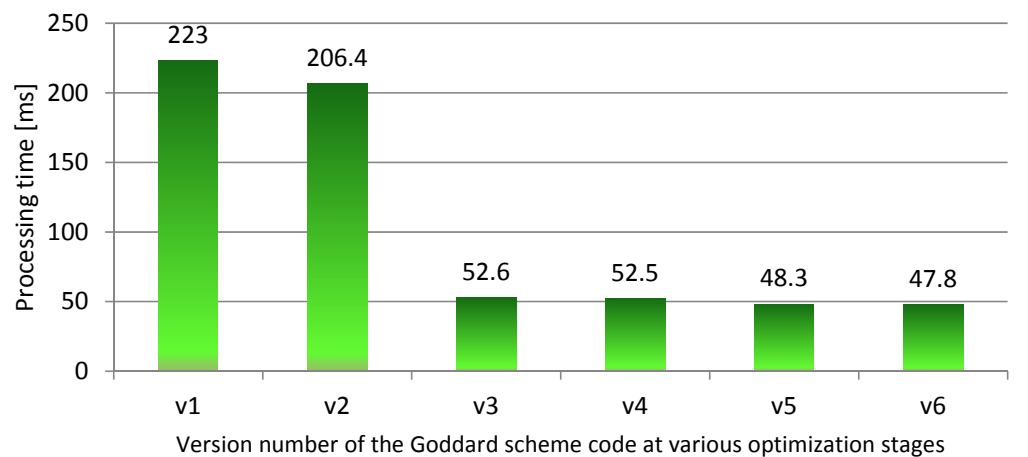

Figure 6. MIC processing time for the Goddard microphysics scheme.

\begin{tabular}{|c|c|}
\hline do $\mathrm{k}=\mathrm{kts}, \mathrm{kte}$ & fluxin $=0$. \\
\hline $\mathbf{q r z}(\mathbf{k})=\mathrm{qr}(\mathrm{i}, \mathrm{k}, \mathrm{j})$ & do $\mathrm{k}=$ max_q,min_q,-1 \\
\hline $\mathbf{d z w}(\mathbf{k})=\operatorname{dz} 8 w(\mathrm{i}, \mathrm{k}, \mathrm{j})$ & $\mathbf{d z w}=\mathrm{dz} 8 \mathrm{w}(\mathrm{i}, \mathrm{k}, \mathrm{j})$ \\
\hline enddo !k & $q r z=q r(i, k, j)$ \\
\hline fluxin $=0$. & fluxout $=$ rhoz $(\mathrm{k}) * \operatorname{vtr}(\mathrm{k}) * \mathbf{q r z}$ \\
\hline do $\mathrm{k}=\max \_\mathrm{q}, \min \_\mathrm{q},-1$ & flux $=($ fluxin-fluxout $) /$ rhoz $(\mathrm{k}) / \mathbf{d z w}$ \\
\hline fluxout $=\operatorname{rhoz}(\mathbf{k}) * \operatorname{vtr}(\mathbf{k}) * \mathbf{q r z}(\mathbf{k})$ & $\mathbf{q r z}=\mathbf{q r z}+$ del_tv*flux \\
\hline flux $=($ fluxin-fluxout $) /$ rhoz $(\mathrm{k}) / \mathbf{d z w}(\mathbf{k})$ & $\mathbf{q r z}=\operatorname{amax} 1(0 ., \mathbf{q r z})$ \\
\hline $\mathbf{q r z}(\mathbf{k})=\mathbf{q r z}(\mathbf{k})+$ del_tv*flux & $\operatorname{qr}(\mathrm{i}, \mathrm{k}, \mathrm{j})=\mathbf{q r z}$ \\
\hline $\mathbf{q r z}(\mathbf{k})=\operatorname{amax} 1(0, \mathbf{q r z}(\mathbf{k}))$ & fluxin=fluxout \\
\hline $\operatorname{qr}(\mathrm{i}, \mathrm{k}, \mathrm{j})=\mathbf{q r z}(\mathbf{k})$ & enddo \\
\hline fluxin=fluxout & if (min_q eq. 1) then \\
\hline enddo & pptrain=pptrain+fluxin*del_tv \\
\hline if (min_q eq. 1) then & else \\
\hline pptrain $=$ pptrain+fluxin*del_tv & $\mathbf{d z w}=\mathrm{dz} 8 \mathrm{w}\left(\mathrm{i}, \mathrm{min} \_\mathrm{q}-1, \mathrm{j}\right)$ \\
\hline else & $\mathbf{q r z}=\mathrm{qr}\left(\mathrm{i}, \min \_\mathrm{q}-1, \mathrm{j}\right)$ \\
\hline $\mathbf{q r z}\left(\min \_\mathbf{q}-1\right)=\mathbf{q r z}\left(\min \_\mathbf{q}-1\right)+\mathrm{del} \_t v^{*} \&$ & $\mathbf{q r z}=\mathbf{q r z}+$ del_tv* \& \\
\hline fluxin/rhoz(min_q-1)/dzw(min_q-1) & fluxin/rhoz(min_q-1)/dzw \\
\hline$q r\left(\mathbf{i}, \min \_q-1, j\right)=q r z\left(\min \_q-1\right)$ & $\operatorname{qr}\left(\mathbf{i}, \min \_\mathrm{q}-1, \mathrm{j}\right)=\mathbf{q r z}$ \\
\hline endif & endif \\
\hline else & else \\
\hline notlast $=$.false. & notlast $=$. false. \\
\hline endif & endif \\
\hline ENDDO & ENDDO \\
\hline
\end{tabular}

Figure 7. The code with $k$-dimension intact for intermediate temporary variables is shown on the left. Code after the dimensions of those variables are reduced to one is shown on the right. Two variables in bold went through the process. 


\begin{tabular}{|c|c|}
\hline $\begin{array}{l}\text { subroutine saticel_s() } \\
\text { do } \mathrm{j}=\mathrm{jts}, \mathrm{jte} \\
\text { do } \mathrm{k}=\mathrm{kts}, \mathrm{kte} \\
\text { do } \mathrm{i}=\mathrm{its}, \mathrm{ite} \\
\quad \ldots \\
\quad \ldots \\
\text { end do } \\
\text { end do } \\
\text { end do } \\
\text { end subroutine saticel_s }\end{array}$ & 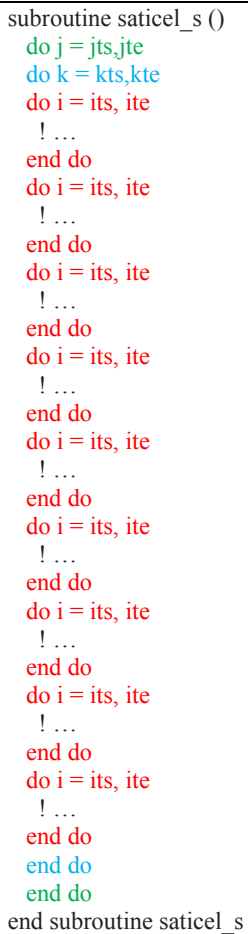 \\
\hline
\end{tabular}

Figure 8. The original non-vectorized code is shown on the left. Vectorized code is shown on the right.

8967

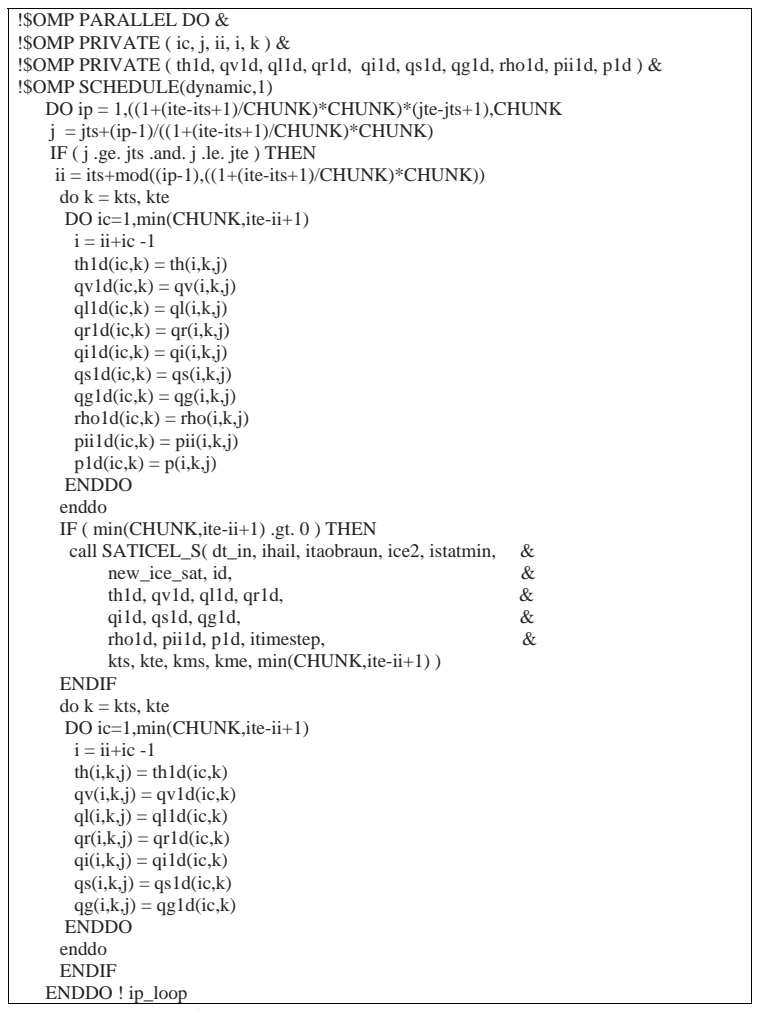

Figure 9. Fortran code for multithreading using OpenMP. Input data is copied to vector sized arrays of CHUNK (16) elements. 


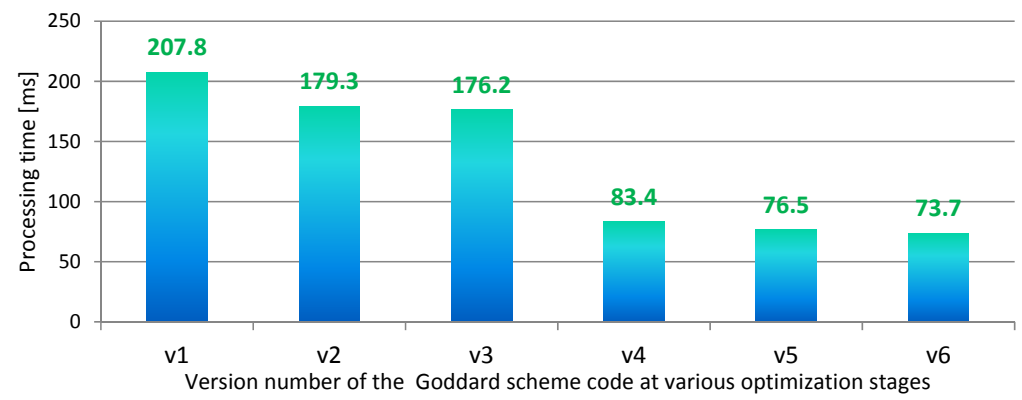

Figure 10. CPU processing time for the Goddard microphysics scheme.

8969

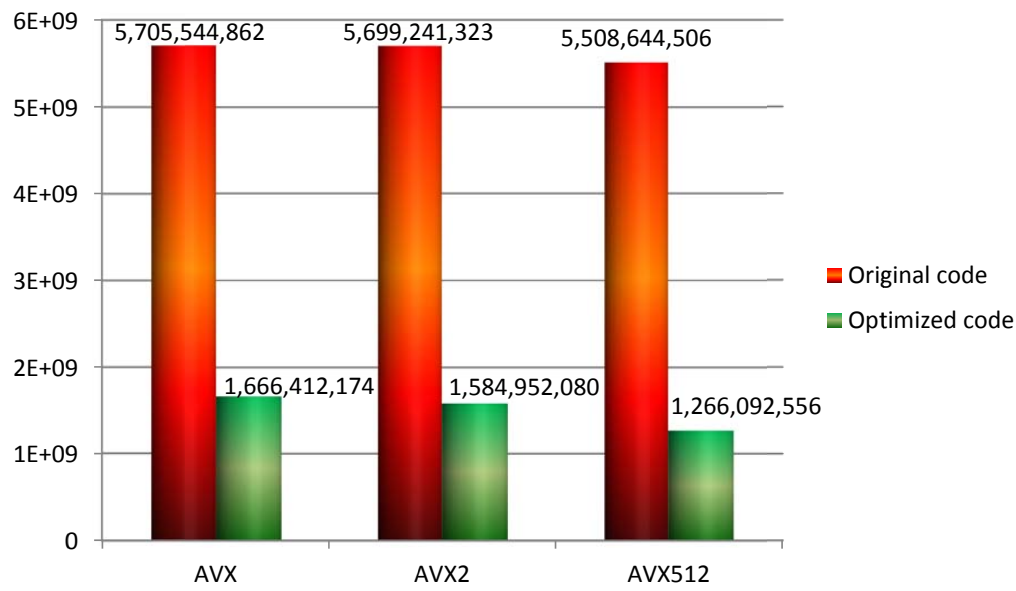

Figure 11. The total number of instruction executed. 


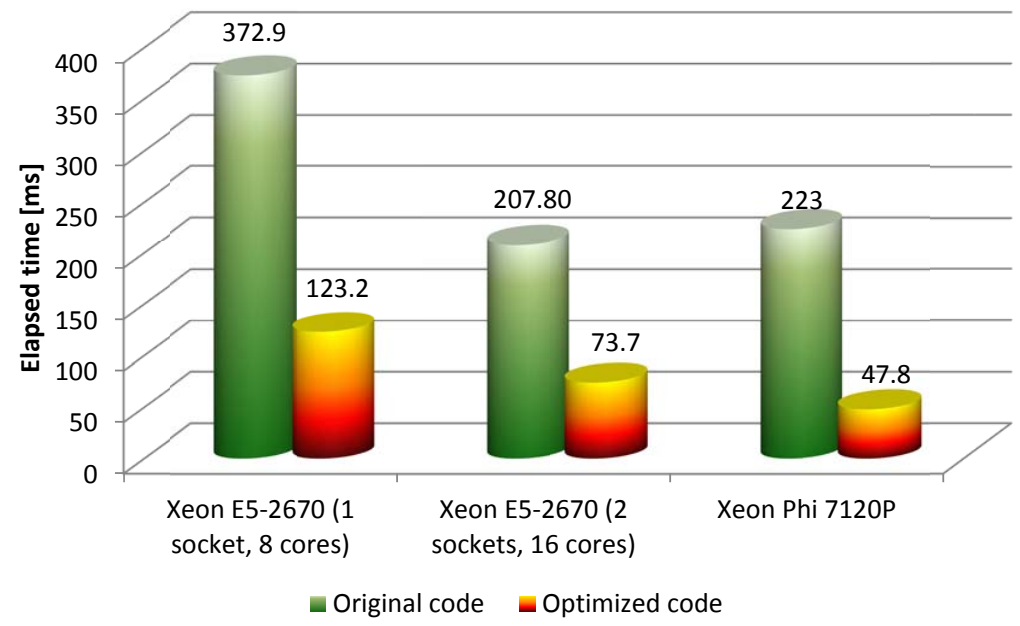

Figure 12. Total elapsed time in milliseconds for execution of Goddard calculation on Xeon CPU with 1 socket and 2 socket configurations and on Intel MIC.

8971

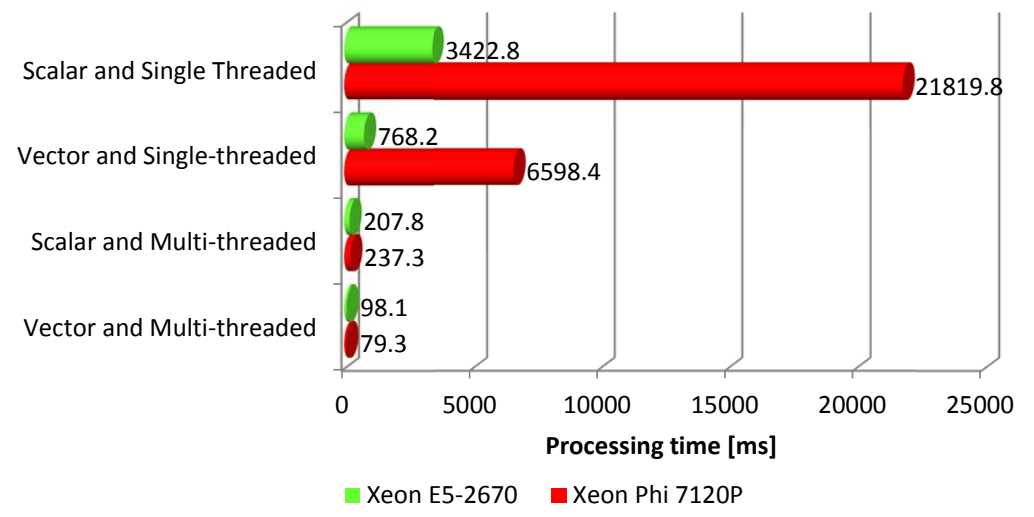

Figure 13. Effects of orthogonal optimization techniques of multi-threading and vectorization on 1 socket Xeon CPU configurations and on Intel MIC. 


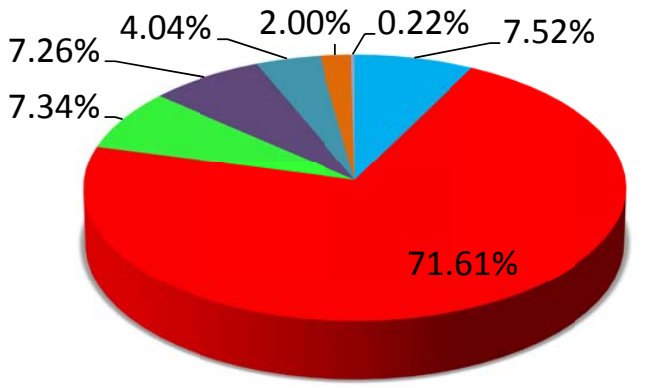

Microphysics: Goddard

Dynamics: ARW

Radiative transfer: rrtm

- Planetary boundary layer: Yonsei

Surface layer: MM5

Figure 14. MIC processing times for the WRF after code optimizations as measured by Intel's profiling tool VTune. 